${ }^{1}$ Departamento de Medicina Familiar, Pontificia Universidad Católica de Chile.

${ }^{2}$ Centro de Educación Médica, Pontificia Universidad Católica de Chile. Santiago, Chile.

32Departamento de Psicología Universidad de Chile.

${ }^{a}$ Doctora en Psicología.

Fuente de Apoyo Financiero: Ninguno.

Recibido el 9 de junio de 2013 , aceptado el 24 de marzo de 2014.

Correspondencia a:

Dra. Philippa Moore Departamento de Medicina Familiar. Escuela de Medicina. Pontificia Universidad Católica de Chile.

Vicuña Mackenna 4686, Macul, Santiago de Chile Tel.: (562) 23548688 moore@med.puc.cl

\section{Logros más alla de los objetivos: evaluación cualitativa de un programa de formación en educación médica}

\author{
PHILIPPA MOORE ${ }^{1}$, LUZ MONTERO $^{1}$, XIMENA TRIVIÑO $^{2}$, \\ MARISOL SIRHAN ${ }^{2}$, LORETO LEIVA,
}

\section{Impact beyond the objectives: a qualitative study of a faculty development program in medical education}

Background: In medical education there has been increasing emphasis on faculty development programs aimed at the professionalization of teaching and increasing students' learning. However, these programs have been shown to have an impact beyond improvement in teaching skills. The medical school of the Pontificia Universidad Católica de Chile (EMUC) has been running a faculty development program (DEM) since 2000. Aim: To explore the perception of graduates on the effects of having participated in DEM on their development as teachers and clinicians. Material and Methods: Using an exploratory, descriptive and qualitative design, the 79 teachers who graduated from DEM from 2004-2008 were sent a questionnaire containing three open questions. Their answers were analyzed using the Constant Comparative Method of Qualitative Analysis of Glaser and Strauss by four researchers. Results: Faculty development, becoming a better clinician, personal development, appreciation of the value of teaching and strengthening of the academic community were the five categories that emerged from the answers. Graduates felt that, besides learning new educational skills, they changed their attitude towards teaching. DEM was perceived as facilitating self-awareness and reflection about the graduates' role as doctors and teachers. The graduates also valued meeting other faculty. Conclusions: Faculty development programs can have an impact far beyond the learning objectives. The planning and design of programs contributes to their wider impact. This should be taken into consideration in the design, planning, and evaluation of faculty development programs. Care should be taken to protect time for participation, reflection and for interaction with other academics.

(Rev Med Chile 2014; 142: 336-343)

Key words: Education, medical; Faculty, medical; Perception; Qualitative research; Teaching.
E $n$ las escuelas de Medicina progresivamente ha ido adquiriendo mayor relevancia la profesionalización de la docencia, dado que el ser un médico experto en el campo clínicocientífico o en investigación no necesariamente asegura ser un educador de excelencia ${ }^{1-3}$. En este escenario se han desarrollado programas de formación en competencias docentes para los profesores de Medicina orientadas a facilitar el aprendizaje efectivo en los estudiantes ${ }^{4}$.

En esta línea, la Escuela de Medicina de la Pontificia Universidad Católica de Chile (EMUC) ha desarrollado un Diplomado en Educación Médica (DEM) para sus académicos. Su propósito es con- 
tribuir a la formación en educación de los docentes de Medicina y de esta manera perfeccionar la docencia de pre y post grado. El DEM está compuesto por cuatro áreas de formación: planificación de la docencia, metodología docente, evaluación de los aprendizajes y desarrollo de habilidades complementarias profesionales (comunicación, ética clínica, medicina basada en la evidencia, etc.).

Tradicionalmente las evaluaciones de la mayoría de estos programas se han realizado de acuerdo al logro de sus objetivos, utilizando una metodología cuantitativa ${ }^{1,4}$. Utilizando ese diseño de investigación, en 2011 fue publicada la evaluación del DEM, basada en el modelo de Kirkpatrick, que mostró un impacto positivo en todos los niveles ${ }^{5}$. Otros estudios han utilizado los métodos cualitativos para explorar con mayor profundidad la perspectiva del académico que participa en estos programas. Estos estudios han mostrado que el realizar un programa de formación docente genera efectos positivos en la vida personal y profesional del graduado, más allá de los objetivos del programa ${ }^{6-8}$.

El objetivo de este estudio fue explorarlos efectos percibidos de haber realizado el DEM en su desarrollo como docentes y médicos clínicos, $\mathrm{y}$ en sus expectativas a largo plazo.

\section{Material y Método}

\section{Diseño}

Diseño descriptivo, exploratorio y de carácter cualitativo con metodología de análisis de contenido. Se define el análisis de contenido como "una técnica de investigación destinada a formular, a partir de ciertos datos, inferencias reproducibles y válidas que puedan aplicarse a su contexto" Este tipo de diseño permite la descripción de los fenómenos al explorar las perspectivas, vivencias y significados de los participantes respecto del tema estudiado $^{10,11}$.

\section{Participantes}

El universo fueron los graduados del DEM. La selección muestral se realizó en base a los siguientes criterios: haberse graduado del DEM en el momento del estudio y haber respondido las tres preguntas abiertas de este estudio.

\section{Recolección y análisis de los datos}

La recolección de la información se realizó a través de una encuesta basada en el Modelo de Kirkpatrick ${ }^{12}$ que se envió el año 2009 a los 91 académicos que se graduaron del DEM entre 2004 y 2008 (vía correo electrónico y postal). El objetivo de la encuesta fue indagar acerca de los efectos percibidos de haber realizado el DEM. La encuesta recogió una serie de datos cuantitativos ya publicados ${ }^{5}$ y además incluía tres preguntas abiertas que son las que se analizan en este estudio.

Las tres preguntas fueron:

1) ¿Cuáles son los efectos más destacados en su desarrollo como médico?

2) ¿Cuáles son los efectos más destacados en su desarrollo como profesor de medicina?

3) ¿Cuáles son sus expectativas a largo plazo?

La participación fue voluntaria y la encuesta fue anónima y se resguardó la confidencialidad a través de un registro independiente con los nombres de los graduados que respondieron la encuesta.

Las respuestas a las preguntas fueron transcritas a una base de datos respetando lo escrito por los participantes, logrando así conservar la descripción de sus experiencias de acuerdo a su óptica, lenguaje y expresiones.

El análisis de contenido fue realizado por cuatro investigadores, inicialmente en forma independiente y luego en conjunto. Se realizó bajo el método comparativo constante de Glasser y Strauss ${ }^{13}$ donde los acontecimientos no se agruparon en categorías predeterminadas, si no que en categorías que fueron emergiendo del análisis de las respuestas de los propios sujetos ${ }^{14}$.

Como fruto de este proceso se obtuvieron las categorías definitivas, es decir aquellas que lograban una adecuada saturación ${ }^{13}$ con sus respectivas frecuencias de codificación. Los comentarios textuales que aquí se presentan fueron seleccionaron por consenso.

Mediante este procedimiento de análisis, se obtuvo una descripción sistemática del contenido manifiesto en las respuestas a las preguntas realizadas. Finalmente, se hizo una triangulación con la información recopilada con la literatura.

\section{Resultados}

La muestra quedó conformada por 79 (87\%) académicos que respondieron las tres preguntas 
abiertas. Las características de los participantes se describen la Tabla 1.

Del análisis del contenido emergieron cinco categorías principales: I) Desarrollo docente; II) Desarrollo como médico clínico; III) Desarrollo personal; IV) Valoración de la docencia y V) Fortalecimiento de la comunidad académica. La Figura 1 muestra las principales categorías con sus respectivas frecuencias de codificación.

\section{Desarrollo docente}

Los docentes perciben haber adquirido un marco conceptual de conocimientos y habilidades relacionadas a la docencia, mencionando todas las áreas desarrolladas en el DEM. También señalan haber adquirido un conjunto de competencias tales como el diseño de programas y de sesiones educativas, el realizar una docencia más participativa, el diseño y aplicación de instrumentos de evaluación del aprendizaje y el desarrollo de habilidades comunicacionales, en especial el feedback.

Mencionan asimismo que están aplicando estas competencias en los distintos escenarios en que se desempeñan, permitiéndoles "asumir el rol del modelo", describiendo que esto les ayuda a profesionalizar la docencia. Ellos destacan que han mejorado su comunicación y su relación con los estudiantes y con otros docentes.

Además, perciben haber experimentado un cambio de actitud frente a la docencia, manifestado como una mayor seguridad frente a los estudiantes y un aumento de la percepción de auto-eficacia en su labor docente, así como un nuevo entusiasmo por la docencia. Usan palabras tales como "amor" y "pasión" por la docencia, valorando su labor docente y reafirmando su rol como profesor, e incluso algunos expresan la aspiración de dedicarse tiempo completo a esta labor.

Describen que el realizar el DEM los animó a tomar nuevos roles docentes, a descubrir la posibilidad de ser un profesor modelo, a continuar el perfeccionamiento docente a través de nuevos cursos del mismo diploma, e incluso, en algunos despertó el interés de realizar investigación en docencia y de especializarse en educación médica.

Citas seleccionadas disponibles en Tabla 2.

\section{Desarrollo como médico clínico}

Los académicos describen que el haber realizado el DEM les ha permitido volver a mirar la forma de relacionarse con sus pacientes. El
Tabla 1. Características demográficas de los graduados del Diplomado en Educación Médica que respondieron las preguntas abiertas de la encuesta $(n=79)$

\begin{tabular}{|llrl|}
\hline \multirow{3}{*}{ Sexo } & Categoría & n & \% \\
& Hombre & 44 & 56 \\
\multirow{3}{*}{ Profesión } & Mujer & 35 & 44 \\
& Médico & 77 & 97 \\
\multirow{2}{*}{ Procedencia } & Odontólogo & 2 & 2,5 \\
& EMUC & 71 & 90 \\
& Otras EM & 8 & 10 \\
\hline
\end{tabular}

EMUC: Escuela de Medicina de la Pontificia Universidad Católica de Chile. EM: Escuela de Medicina.

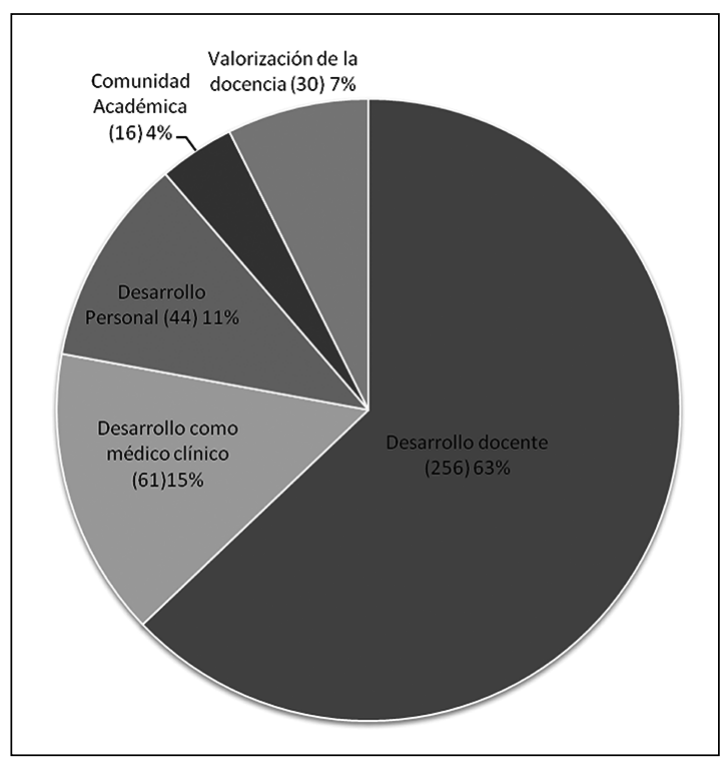

Figura 1. Categorías emergentes con el número de comentarios analizados y su distribución porcentual.

aplicar las habilidades aprendidas, especialmente las comunicacionales, les ha ayudado a realizar una atención más centrada en la persona. Emerge una visión más integral de la medicina, un "mayor desarrollo de la perspectiva humanista de la medicina". Lo aprendido en relación con la educación participativa de adultos los hace sentirse más competentes al educar a sus pacientes: "al mejorar en lo docente... mejora la entrega de información a los pacientes". También destacan la aplicación de los conocimientos adquiridos en los cursos de ética clínica y de medicina basada en evidencia 
Evaluación cualitativa de programa de educación médica - P. Moore et al

Tabla 2. Citas textuales seleccionadas de la categoría

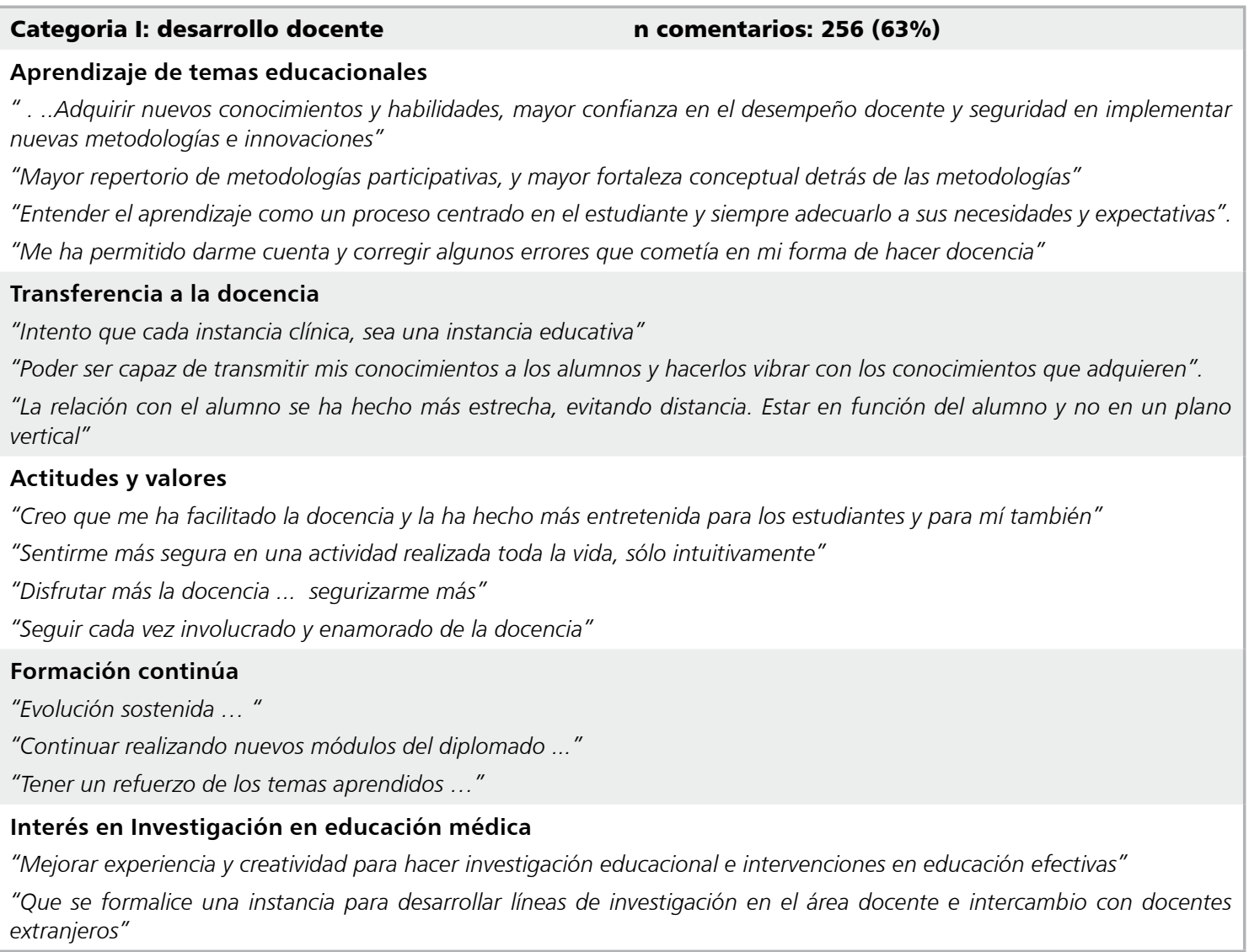

en su quehacer clínico cotidiano. Por último, hay un replanteamiento de actitudes en relación a su quehacer clínico: la motivación de "ser un médico más competente" y de hacer esfuerzos para lograr un mejoramiento continuo.

\section{Desarrollo personal}

El haber realizado el DEM es percibido como una instancia de reflexión y crecimiento personal que permite a los docentestener un mayor auto-conocimiento. Describen que algunas de las habilidades adquiridas son utilizadas más allá del ámbito clínico o docente, destaca el caso de las habilidades relacionales y su aplicación en la vida cotidiana. Emerge una visión más integral de la vida, un desarrollo de la capacidad de reflexión sobre la acción y una percepción de mayor satisfacción personal y seguridad en sí mismos.

\section{Valoración de la docencia}

Los académicos perciben que el DEM ha contribuido a profesionalizar la docencia en la EMUC, la cual ya no es vista como algo innato, sino como una actividad que se puede perfeccionar. Los egresados descubren o reafirman una "convicción personal" de que "es posible y deseable potenciar las capacidades de cada uno de los académicos". También el DEM ha permitido crear un lenguaje común sobre temas docentes y ha aumentado la valoración de la docencia en la institución tanto por los académicos como por los estudiantes.

\section{Fortalecimiento de la comunidad académica}

Los egresados valoraron la oportunidad de conocer a otros docentes de la comunidad académica y compartir con colegas que conocían "solamente en los pasillos", lo que les genera una 


\section{Tabla 3. Citas textuales seleccionadas de categorías II a V}

\section{Categoría II: desarrollo como médico clínico \\ n comentarios: $61(15 \%)$}

"Mejoría en la relación médico-paciente, insta a ser mejor persona, aprender a escuchar, interpretar otros lenguajes, a respetar diferentes culturas"

"Mejorar mi rol como educador del paciente y la familia, cultivar una relación médico-paciente más efectiva, reafirmar la forma profesional integral y su beneficio en el cuidado del paciente"

"Complementar el desarrollo profesional con la práctica de medicina basada en evidencia en un marco ético que motive la docencia e investigación".

"Replantearme en muchas ocasiones la manera en que realizo mi quehacer médico cotidiano, en la relación con mis pacientes y sus padres"

\section{Categoría III: desarrollo personal}

n comentarios: $44(11 \%)$

"Aprender a preguntarme día a día de mis habilidades y competencias"

"Me ha permitido darme cuenta y corregir algunos errores que cometía en mi forma de hacer docencia"

"Satisfacción personal......"

"....insta a ser mejor persona, aprender a escuchar, interpretar otros lenguajes, a respetar diferentes culturas"

"Abrir horizontes de desarrollo, crecimiento y formación"

"Honestamente, siento que ha sido una maravillosa oportunidad de superación"

\section{Categoría IV: valoración de la docencia \\ n comentarios: $30(7 \%)$}

"La adquisición de conciencia sobre la gran responsabilidad que implica la docencia para con los futuros pacientes de mis alumnos"

"El reconocimiento por parte de los alumnos de mi desempeño docente"

"Sentir valorada la calidad docente....."

"Legitimar el tema de la docencia como una competencia necesaria para el adecuado cumplimiento de las actividades docentes"

"Generar una cultura de valoración de la capacitación docente entre el cuerpo de académicos"

"Rescatar la labor docente como que requiere conocimiento, y no es algo que fluye, y que puede hacer cualquier persona" Categoría V: fortalecimiento de la comunidad académica

n comentarios: $16(4 \%)$

"Compartir con otros de la escuela, de ámbitos muy diversos, contribuyendo a sentirme más integrada y parte de la facultad, la cual ahora valoro y conozco mejor"

"Conocí mucha gente que sólo había visto en los pasillos del hospital, esto sirve mucho para interrelacionarse y formar equipos de trabajo"

mayor sensación de pertenencia a la institución. Ven en estas nuevas interacciones una oportunidad para formar equipos de trabajo y tener más sentido de Facultad.

Citas seleccionadas de las categorías II a V se encuentran en la Tabla 3.

\section{Discusión}

Los resultados de este estudio muestran que los docentes perciben que la realización del DEM tiene efectos positivos en múltiples áreas. En pri- mer lugar destaca la adquisición de conocimientos y habilidades en docencia en concordancia con los objetivos del programa, en especial, en las áreas de comunicación y feedback, evaluación del aprendizaje, planificación y metodologías participativas. Además los académicos perciben que no solamente aprenden, sino que logran transferir estos conocimientos y habilidades a su labor docente. Esto corrobora los hallazgos previamente publicados, en los cuales $93 \%$ de los encuestados respondió estar de acuerdo o muy de acuerdo en haber logrado transferir estos aprendizajes a su actividad docente cotidiana ${ }^{5}$. 
En segundo lugar destaca la percepción de que el realizar el DEM genera un cambio de actitud positivo frente a la docencia. Los egresados describen una mayor motivación y seguridad al hacer docencia y valoran la actividad docente como algo relevante en su actividad académica. También descubren que la docencia es algo que requiere capacitación y se motivan por seguir aprendiendo e investigando en el área de educación médica. Esto también confirma los resultados cuantitativos publicados anteriormente, donde 93,8\% de los encuestados respondió estar de acuerdo o muy de acuerdo con que el DEM ha contribuido a tener una actitud favorable hacia la docencia ${ }^{5}$. Estos resultados contrastan con las creencias encontradas en aquellos docentes que optan por no capacitarse en docencia, los cuales perciben la docencia como algo innato y que no requiere mayor capacitación ${ }^{15,16}$.

En tercer lugar sorprende que el realizar este diplomado tiene efectos más allá de su propósito, el que se observa en diversas áreas: crecimiento como persona o "integral", el aumento del sentido de pertenencia a la institución y la valoración de la docencia por parte de la institución, así como el fortalecimiento de la comunidad académica. Estos hallazgos confirman lo encontrado por otras investigaciones: Knight concluye que programas de formación de docentes en educación médica pueden tener efectos amplios y duraderos en el desarrollo intra e interpersonal, así como también en la carrera docente ${ }^{6}$. Lieff plantea que la capacitación docente puede tener un impacto en los "valores humanistas" de los académicos y puede alentar la reflexión sobre el sentido de ser docente y sobre la búsqueda de la realización como profesional ${ }^{17}$, lo cual contribuye al desafío de retener alos académicos y consolidar un grupo altamente motivado con un sentido de pertenencia a su institución ${ }^{18,19}$.

\section{¿Cuáles son los elementos que explican estos efectos más allá de los objetivos?}

La respuesta a esta pregunta surge desde la historia del DEM, ya que en sudiseño se siguieron los pasos recomendados por expertos ${ }^{20}$. Primero se conformó un equipo de académicos liderado por un profesor con experiencia y liderazgo. Luego, utilizando la metodología de la educación de adultos $^{21}$, se realizó un análisis de las necesidades de aprendizaje de los futuros alumnoslo que permitió afinar los objetivos y contenidos, realizándose en paralelo una reflexión acerca del sentido de la pedagogía y de lo que se quería impartir ${ }^{22}$.

A continuación, se diseñó el programa utilizando varios de los elementos claves para una formación docente exitosa ${ }^{16,23}$ : propósito y objetivos que responden a las necesidades institucionales, formato longitudinal, diseño integral, voluntario, conducción por profesores expertos en docencia, metodologías centradas en el aprendizaje y una evaluación periódica. También hubo otros aspectos que lo hicieron atractivo para los académicos: programa flexible y gratuito, dentro del horario de trabajo, contenidos adecuados a las necesidades de los participantes, respaldo y reconocimiento por las autoridades, etc. Por otra parte, el hecho de que los alumnos pertenecieran a diferentes departamentos permitió el intercambio y socialización con otros académicos, aspecto especialmente valorado por nuestros egresados y por diversos grupos de académicos en otros estudios ${ }^{6,24-26}$.

Por último, la metodología participativa empleada, con trabajo en pequeño grupo y énfasis en la discusión, tal como describen los expertos, facilitó la reflexión sobre problemas de la propia experiencia, promoviendoel aprendizaje en áreas como el profesionalismo, así como el desarrollo personal ${ }^{27}$, y brindó un espacio para profundizar sobre el sentido de la carrera académica e incluso sobre el sentido de la vida ${ }^{28}$. Algunas de estas experiencias de reflexión más profunda surgidas en este diplomado, ya han sido publicadas ${ }^{29,30}$.

La metodología utilizada en este estudio, cuyo análisis está fundado en la información levantada a partir de preguntas abiertas enviadas al universo completo de los egresados del DEM, permite abrir ventanas para entender en forma más amplia los efectos de un programa de este tipo ${ }^{31}$. Sin embargo, es importante destacarque la participación fue voluntaria y que las respuestas a las preguntas abiertas fueron espontáneas, por lo tanto no se puede asumir que las categorías mencionadas con menos frecuencia tienen menor importancia. Si se hubiese encuestado a todos los egresados sobre cada categoría es probable que el número de respuestas en cada categoría hubiese sido mayor.

Si bien el auto-reporte de los estudiantesdocentes puede presentar un sesgo de memoria selectivo; sería importante en futuros estudios relacionar estos resultados con conductas observables en su docencia o en la facilitación del aprendizaje 
de sus estudiantes. No obstante, el auto-reporte y la riqueza de los comentarios emitidos en este estudio constituyen también una de sus fortalezas, posibilitando el conocimientode las percepciones y experiencias de los docentes como estudiantes.

Una limitación de este estudio podría ser que cuatro de los cinco investigadores, son profesores del DEM, lo cual podría producir un sesgo en la interpretación de los datos.

Para profundizar más en los resultados proponemos una segunda fase con entrevistas en profundidad o grupos focales a los egresados.

\section{¿Qué recomendaciones para el futuro de la formación docente pueden derivarse de estos resultados?}

Dado que este tipo de programa puede tener un impacto en el desarrollo académico y personal de los participantes que va más allá de los objetivos de aprendizaje, los directores de programas de desarrollo docente deberían considerar estos efectos en su diseño y evaluación. Algunos autores proponen añadir a los objetivos metas más amplias y ambiciosas tales como el fomentar liderazgos docentes, crear oportunidades para la investigación educacional, apoyar con mentorías a los académicos jóvenes, estimular el compromiso con la labor académica y los lazos de pertenencia e identidad con la cultura y los valores de la comunidad académica ${ }^{8,24-26}$. Pololi va más allá y propone un "cambio de paradigma" en el cual el aprendizaje afectivo, que incluye la capacidad de auto-reflexión sobre el sentido de la propia docencia y sobre surealización como profesional, debiera ser la meta de formación docente para lograr una "experiencia transformacional" 32 . Independientemente de si este aprendizaje "afectivo" queda plasmado en los objetivos o simplemente queda en el curriculum oculto, los directores de programas deben resguardar una metodología participativa, con espacios de reflexión, intercambio entre académicos y espacios sociales como el café.

El financiamiento de tales programas puede verse facilitado por el hecho de que las autoridades van a percibir efectos positivos no solamente en los egresados sino también en el ambiente académico e institucional. Finalmente, queda planteado como un desafío a la investigación en educación médica evaluar el impacto de los programas de formación docente sobre el aprendizaje de los estudiantes de pre y post grado.
Agradecimientos: A los Dres. José Chianale, Rodrigo Moreno y Vicente Valdivieso, por ser los promotores de la iniciativa que llevó a la creación del Diplomado en Educación Médica en la EMUC, y especialmente al Dr. Valdivieso por haber liderado su desarrollo. También a los 30 académicos que se han constituido como "profesores de profesores" a lo largo de estos años impartiendo cursos y a los 79 egresados quienes respondieron a las preguntas de la encuesta.

\section{Referencias}

1. Triviño X, Sirhan M, Moore P, Reyes C. [Faculty development for clinicaI teachers in medicine]. Rev Med Chile 2009; 137 (11): 1516-22.

2. Steinert Y. Mapping the teacher's role: the value of defining core competencies for teaching. Med Teach 2009; 31 (5): 371-2.

3. Rosselot E. [Looking for the physicians that our countries need: emphasis on communication and training of academics]. Rev Med Chile 2003; 131 (3): 331-7.

4. Steinert Y, Mann K, Centeno A, Dolmans D, Spencer J, Gelula M, et al. A systematic review of faculty development initiatives designed to improve teaching effectiveness in medical education: BEME Guide No. 8. Med Teach 2006; 28 (6): 497-526.

5. Triviño X, Sirhan M, Moore P, Montero L. [Impact of a diploma on medical education in a medical school in Chile]. Rev Med Chile 2011; 139 (11): 1508-15.

6. Knight AM, Carrese JA, Wright SM. Qualitative assessment of the long-term impact of a faculty development programme in teaching skills. Med Educ 2007; 41 (6): 592-600.

7. Lieff SJ. Perspective: The missing link in academic career planning and development: pursuit of meaningful and aligned work. Acad Med 2009; 84 (10): 1383-8.

8. Lown BA, Newman LR, Hatem CJ. The personal and professional impact of a fellowship in medical education. Acad Med 2009; 84 (8): 1089-97.

9. Krippendorff K. Metodología de análisis de contenido: teoría y práctica. Editorial Paidós; 1990.

10. Miles MB, Huberman AM, Saldana J. Qualitative Data Analysis: A Methods Sourcebook: Third Edition: Third. Thousand Oaks: SAGE Publications, Inc; 2014.

11. Flick U. An Introduction to Qualitative Research. SAGE Publications Ltd; 2009.

12. Kirkpatrick DL. Evaluating Training Programs: The Four Levels. Berrett-Koehler; 1994.

13. Corbin J, Strauss A. Basics of Qualitative Research: Tech- 
Evaluación cualitativa de programa de educación médica - P. Moore et al

niques and Procedures for Developing Grounded Theory. Third Ed. SAGE Publications, Inc; 2007.

14. Flores Guerrero R. Observando observadores: una introducción a las técnicas cualitativas de investigación social. Santiago: Eds. Universidad Católica de Chile; 2009.

15. Montero L, Triviño X, Sirhan M, Moore P, Leiva L. [Barriers for faculty development in medical education: a qualitative study]. Rev Med Chile 2012; 140 (6): 695702.

16. Steinert Y, Mann K V. Faculty development: principles and practices. J Vet Med Educ 2006; 33 (3): 317-24.

17. Lieff S, Baker L, Mori B, Egan-Lee E, Chin K, Reeves S. Who am I? Key influences on the formation of academic identity within a faculty development program. Med Teach 2012; 34 (3): e208-15.

18. AAMC. The long-term retention and attrition of U.S. medical faculty. AAMC Analysis in Brief. 2008.

19. Ioannidis JPA. Academic medicine: the evidence base. BMJ 2004; 329 (7469): 789-92.

20. McLean M, Cilliers F, Van Wyk JM. Faculty development: yesterday, today and tomorrow. Med Teach 2008; 30 (6): 555-84.

21. Vella J. On Teaching and Learning: Putting the Principles and Practices of Dialogue Education into Action. First. Jossey-Bass; 2008.

22. Torralba Rosello F. Pedagogía del Sentido. Madrid: PPC; 2005.

23. Gruppen LD, Simpson D, Searle NS, Robins L, Irby DM, Mullan PB. Educational fellowship programs: common themes and overarching issues. Acad Med 2006; 81 (11):
990-4.

24. Pololi LH, Knight SM, Dennis K, Frankel RM. Helping medical school faculty realize their dreams: an innovative, collaborative mentoring program. Acad Med 2002; 77 (5): 377-84.

25. Armstrong EG, Doyle J, Bennett NL. Transformative professional development of physicians as educators: assessment of a model. Acad Med 2003; 78 (7): 702-8.

26. Steinert Y, Nasmith L, McLeod PJ, Conochie L. A teaching scholars program to develop leaders in medical education. Acad Med 2003; 78 (2): 142-9.

27. Goldstein EA, Maestas RR, Fryer-Edwards K, Wenrich MD, Oelschlager A-MA, Baernstein A, et al. Professionalism in medical education: an institutional challenge. Acad Med 2006; 81 (10): 871-6.

28. Vella J. Learning to Listen, Learning to Teach: The Power of Dialogue in Educating Adults. Revised ed. San Francisco: Jossey-Bass; 2008.

29. Walker MR, Zúñiga D, Triviño X. [Narrative and faculty development: results of a five years experience with a creative writing workshop]. Rev Med Chile 2012; 140 (5): 659-66.

30. Moore P, Gómez G, Kurtz S. [Doctor-patient communication: one of the basic competencies, but different]. Aten Primaria 2012; 44 (6): 358-65.

31. Lingard L, Kennedy TJ. Qualitative Research in Medical Education. ASME; 2007.

32. Pololi LH, Frankel RM. Humanising medical education through faculty development: linking self-awareness and teaching skills. Med Educ 2005; 39 (2): 154-62. 